\title{
Tricuspid regurgitation as a result of Chiari network attachment
}

\author{
Jen-Ping Chang, MD
}

A 53-year-old man with a 2-year-history of severe mitral re-
gurgitation and moderate tricuspid regurgitation (TR) was
admitted for elective valvular reparative surgery. Previous
2-dimensional echocardiography had revealed severe mitral
regurgitation as a result of myxomatous prolapse of postero-
medial commissural scallop and moderate concentric TR.
Cardiac catheterization confirmed normal coronary arteries
with moderate pulmonary hypertension. The mitral valve
was repaired with posteromedial commissural sliding plasty
and ring annuloplasty. Subsequently, the right atrium was
opened, and the TR was found to be caused by an abnormal
Chiari network attachment to the septal leaflet, which re-
sulted in persistent prolapse of the leaflet and concentric
TR (Figure 1). The Chiari network was excised, and the mod-
erately dilated tricuspid annulus was repaired with ring annu-
loplasty. Postoperative recovery was uneventful. Four years
after the operation, follow-up 2-dimensional echocardiogra-
phy showed a competent mitral valve and trivial TR.
The Chiari network, a congenital reticulated structure
from the right valve of the sinus venosus (eustachian valve)
connecting to various part of the right atrium, is seen in
$1.3 \%$ to $4 \%$ of autopsy subjects. Generally, the structure
is thought to be clinically insignificant; however, it has
been reported to be associated with patent foramen ovale
with intense right-to-left shunting and with atrial septal an-
eurysm. The Chiari network has also been reported to be

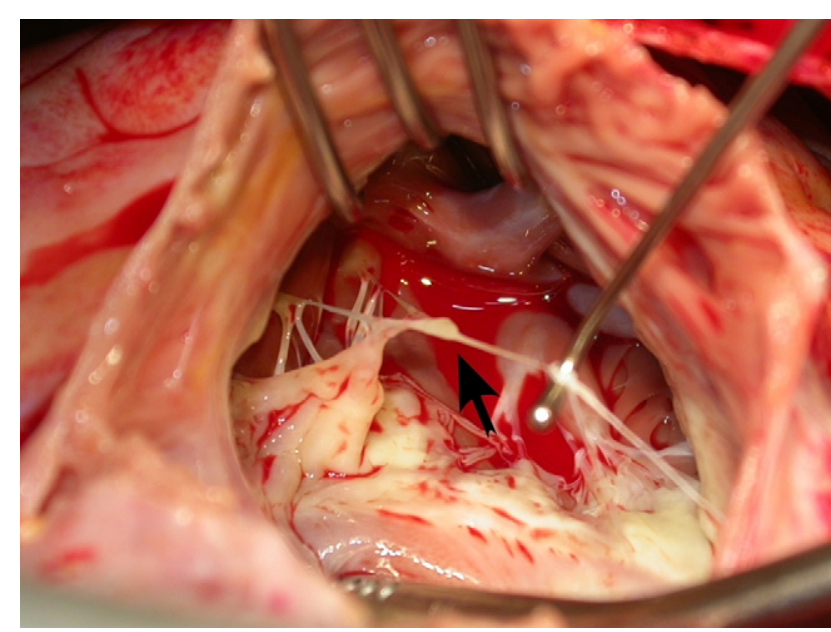

FIGURE 1. Intraoperative photograph showing an abnormal Chiari network attachment to the septal leaflet (arrow), which resulted in persistent prolapse of the leaflet and concentric tricuspid regurgitation.

involved in the pathogenesis of arterial embolism, infectious endocarditis, supraventricular arrhythmia, and catheter entrapment.

This represents the first reported case of TR caused by Chiari network attachment and shows an image of this unique anomaly.

\footnotetext{
From the Division of Thoracic and Cardiovascular Surgery, Department of Surgery, Kaohsiung Chang Gung Memorial Hospital, Chang Gung University College of Medicine, Taiwan, Republic of China.

Disclosures: Author has nothing to disclose with regard to commercial support.

Received for publication June 22, 2011; accepted for publication June 28, 2011; available ahead of print July 25, 2011.

Address for reprints: Jen-Ping Chang, MD, Division of Thoracic and Cardiovascular Surgery, Department of Surgery, Kaohsiung Chang Gung Memorial Hospital, Chang Gung University College of Medicine, 123, Tapei Rd, Niaosung District, Kaohsiung City, Taiwan, Republic of China 833 (E-mail: c9112772@adm.cgmh. org.tw).

J Thorac Cardiovasc Surg 2012;143:235

$0022-5223 / \$ 36.00$

Copyright (C) 2012 by The American Association for Thoracic Surgery

doi:10.1016/j.jtcvs.2011.06.034
} 